\title{
Transforming Growth Factor-Beta Superfamily
}

National Cancer Institute

\section{Source}

National Cancer Institute. Transforming Growth Factor-Beta Superfamily. NCI

Thesaurus. Code C20455.

\begin{abstract}
A family of structurally-related cytokines that bind to their cognate serine/threonine kinase receptors as homodimers or heterodimers linked by a single disulfide bond. These proteins play a role in embryonic development, hematopoiesis, tissue repair and extracellular matrix remodeling.
\end{abstract}

